\title{
Former les étudiants scientifiques à l'épistémologie par la pratique de la réplication d'expériences historiques
}

\author{
Jérôme Fatet ${ }^{\mathrm{a}}$ \\ FRED (EA6311), Université de Limoges, ESPE du Limousin, France
}

\begin{abstract}
Résumé. Nous présentons ici les travaux menés à l'IUFM du Limousin permettant aux étudiants de manipuler des appareils anciens et de réaliser avec ceux-ci des réplications d'expériences historiques. Cette activité permet, au delà de la construction d'une culture historique, d'explorer la portée et les limites du savoir scientifique dans la manière dont il a été élaboré. Elle leur offre ainsi l'occasion de s'interroger sur le sens que l'on peut - ou que l'on a pu - donner à un résultat expérimental et sur les conditions dans lesquelles il a été discuté, rejeté ou validé. Cette approche leur permet d'aborder les questions épistémologiques fondamentales de manière concrète et ainsi d'apporter du sens aux cours théoriques d'épistémologie.
\end{abstract}

\begin{abstract}
We present here a work lead at the IUFM du Limousin. Its aim is to mentor students with manipulating olden apparatus untaking replications of historical experiments. Beyond building historical culture, this process - in the manner it has been elaborated enables students to explore the impact and the limits of scientific knowledge. This activity can also give them the ability of wondering both of the meaning to give - or that as been given - to an expérimental result and on the conditions it has been discussed, rejected or confirmed. This approach leads them to broach fundamental epistemological questionning by manipulating, in order to make sense to the the academic episemology.
\end{abstract}

Afin de comprendre les motifs de la mise en place de l'expérimentation que nous allons décrire dans cet article il nous faut tout d'abord préciser le contexte institutionnel à la fois national et local qui a permis la mise en ouvre de ce travail.

Dès 2008, la mise en place des formations masterisées pour la préparation aux concours de l'enseignement secondaire a pris en compte l'importance de l'enseignement de l'épistémologie pour les futurs enseignants de sciences physiques. Dans ce contexte, une place importante a été accordée à ces contenus dans la formation des futurs enseignants de sciences physiques à l'Université de Limoges. Parallèlement, la mise en œuvre de travaux encadrés de recherche, menés sur les deux années de master par ces mêmes étudiants nous a offert la possibilité d'explorer avec eux la mise en œuvre de questions épistémologiques dans le cadre de projets longs et durables.

\footnotetext{
a e-mail : jerome.fatet@unilim.fr
}

This is an Open Access article distributed under the terms of the Creative Commons Attribution License 4.0, which permits unrestricted use, distribution, and reproduction in any medium, provided the original work is properly cited. 
Les contenus visés dans ces enseignements ont été identifiés en fonction de besoins et des nécessités d'un enseignement de la physique et de la chimie efficace au collège comme au lycée, prenant en compte le renouvellement des programmes ${ }^{2}$ du collège puis du lycée dans lesquelles les questions épistémologiques liées au développement des sciences et à la validité des concepts et des modèles mis en jeu peuvent être discutés et analysés. Toutefois, s'il devient possible, et pertinent en terme de respect des programmes, d'aborder de tels contenus, cela s'avère périlleux sans une solide formation initiale. Pour permettre à ces futurs enseignants de développer ce type d'approche, les contenus que nous avons choisis de développer au cours des deux années de Master Métier de l'Enseignement de l'Education et de la Formation (MEEF) à l'Université de Limoges concernent en particulier le rôle de l'expérience dans l'élaboration et la transmission des savoirs, le statut des instruments pour l'exploration expérimentale ainsi que le sens du résultat numérique lors de l'exploitation d'une expérience. L'enjeu de ce type d'enseignement est d'accompagner les étudiants dans l'évolution de leurs conceptions sur la nature même des sciences pour agir sur les modes d'enseignement qu'ils développeront en tant que professionnels. Ces futurs enseignants ainsi formés doivent permettre à leurs élèves de comprendre dès le début de leur formation scientifique les spécificités de ce savoir. Dans ce but, l'enseignement d'épistémologie a été construit à l'Université de Limoges en étroite relation avec les contenus de didactique des sciences. Ainsi, nous pensons pouvoir tisser des liens entre l'élaboration des savoirs dans le processus de découverte scientifique et l'élaboration de savoir dans les processus d'acquisition des connaissances par les élèves.

Après trois ans d'expérimentation de ce type d'enseignement le constat que nous avons pu tirer concernant l'efficacité de ce travail nous apparaît mitigé. Si la place accordée à ces contenus dans la formation est importante, et si l'intérêt que les étudiants portent à ceux-ci est véritable, leur capacité à les transposer à l'analyse de leur propre pratique scientifique et au contexte concret de l'enseignement semble faible. L'enjeu est pourtant, pour nous, plutôt de leur permettre d'utiliser quotidiennement ces savoirs que de leur offrir une compétence réellement académique en épistémologie des sciences.

Face à ce constat, il nous a semblé nécessaire de trouver des modes de formation différents, permettant d'atteindre ce but. Nous avons donc choisi de mettre en œuvre une stratégie semblant permettre d'atteindre mieux nos objectifs en tentant de répondre à la question : une approche expérimentale de certaines questions centrales de l'épistémologie des sciences ne pourrait-elle pas résoudre cette difficulté de transposition de savoirs académiques vers la pratique quotidienne de l'enseignement des sciences?

\section{Cadre théorique}

\subsection{Un projet expérimental}

Ce projet d'expérimentation menée auprès des étudiants, futurs enseignants de sciences physiques vise donc à leur permettre de comprendre au quotidien les spécificités de l'élaboration des savoirs scientifiques, en particulier pour ce qui concerne le rôle et la place de l'expérience dans la construction des savoirs. La mise en œuvre d'enseignements académiques ne permettant vraisemblablement pas la structuration d'un processus réflexif, il nous a semblé pertinent d'envisager de les leur faire découvrir par eux-mêmes, en les impliquant dans un pratique scientifique réelle, concrète et encadrée.

Cette distinction, que l'on pourrait qualifier de manière caricaturale de « différence entre apprendre et comprendre» un contenu n'est pas récente. Et l'intérêt de l'expérimentation pour l'appropriation des

\footnotetext{
2 On peut lire, dans le préambule des derniers programmes de Physique-Chimie de Terminale S : «Les activités proposées aux élèves [...] doivent les conduire à s'interroger de manière critique sur la valeur scientifique des informations [...] ». Ministère de l'Education Nationale, de la Jeunesse et de la vie Associative, Programme de l'enseignement spécifique et de spécialité de physique-chimie, Bulletin Officiel spécial $N^{\circ} 8$ du 13.10.2011, p1.
} 
contenus a été identifié, pour ce qui concerne les sciences expérimentales, depuis le milieu du XXe siècle. Thomas Kuhn en donne une analyse dès 1970 :

[...] apprendre une théorie est un processus qui dépend de l'étude de ses applications et de la résolution de problèmes pratiques, aussi bien avec un papier et un crayon qu'avec des instruments de laboratoire. $\mathrm{Si}$, par exemple, un individu étudiant la dynamique d'après les lois de Newton découvre un jour le sens de termes comme force, masse, espace et temps, ce sera moins parce qu'il en aura trouvé dans son texte des définitions (incomplètes mais souvent utiles) que parce qu'il aura observé en y participant lui-même, l'application de ces concepts à la solution de problèmes. ${ }^{3}$

Puisque cette distinction a pu être opérée et validée pour ce qui concerne l'apprentissage des sciences expérimentales il semble intéressant de se demander si elle ne pourrait pas être efficace pour la structuration des savoirs en épistémologie de ces sciences expérimentales.

\subsection{Vers la réplication d'expériences historiques}

Afin d'explorer cette piste, nous avons choisi d'expérimenter avec les étudiants la mise en œuvre de réplications d'expériences historiques dans le but de faire émerger les spécificités épistémologiques qu'elles peuvent soulever. Nous souhaitons ainsi qu'ils parviennent spécifiquement à cerner les particularités que présente l'invention même de l'expérimentation, les processus d'acquisition des résultats expérimentaux, les choix opérés dans le tri de ces résultats, ainsi que les modes de conservation de ceux-ci.

Le choix de mener ce travail sur des expériences historiques présente deux avantages. Tout d'abord il permet d'éviter l'écueil de la controverse en cours. Si controverse il y a eu, et c'est souvent le cas, elle est résolue. La vraisemblance, l'étendue et les limites des savoirs mis en jeu et leur stabilité sont donc connues. Ceci nous permet de nous dégager des débats du moment. Ensuite, les thématiques étudiées sont le plus souvent considérées comme valide voire probante par la science actuelle. Accompagner les étudiants dans une réflexion sur l'élaboration et la validation de ces « preuves » peut alors permettre l'apparition de réflexion sur le sens de cette expression dans la recherche scientifique actuelle, et dans l'enseignement des sciences expérimentales dans lequel ce concept est souvent manipulé sans précaution.

Nombreux sont les historiens des sciences qui mettent en œuvre une approche de l'enseignement de l'histoire des sciences et de l'enseignement de certains contenus scientifiques par la réplication d'expériences historiques ${ }^{4}$. La pertinence de cette démarche semble dorénavant acceptée. L'utilisation que nous proposons ici de ces outils pour une approche de l'enseignement de l'épistémologie, elle, n'a pour le moment pas été explorée.

\section{Mise en œuvre des réplications 5}

\subsection{Mesurer et enregistrer}

Les expériences dites historiques sont nombreuses dans l'enseignement des sciences au collège ou lycée. Mentionnées dans les programmes officiels ${ }^{6}$, elles sont aussi présentes dans de nombreux manuels

\footnotetext{
${ }^{3}$ Kuhn T. (1983) La structure des révolutions scientifiques, trad.. Laure Meyer, Champs/Flamarion n ${ }^{\circ} 115$, p76.

${ }^{4}$ Un ouvrage traitant de ces questions est paru en 2011 : Heering P., Wittje R. (2011) Learning by doing, Franz Steiner Verlag.

5 Dans la suite de ce texte, nous illustrerons certains de nos propos en faisant référence à l'une des réplications que nous avons mise en œuvre avec nos étudiants : la production de rayons X. Les points que nous présenterons n'auront que valeur d'exemple. Nous ne détaillerons pas ici l'intégralité du travail de réplication, puisque ce n'est pas l'objet de l'article.

${ }^{6}$ L'expérience du prisme mobile d'Arago dans le programme de Terminale S par exemple : opt.cit.
} 
scolaires. Qu'elles y soient décrites ou que l'on propose aux enseignants de les réaliser, elles sont très éloignées, la plupart du temps, de l'expérience d'origine à laquelle elles font référence. L'intérêt historique et scientifique de la présentation de tels objets d'enseignement est semble-t-il reconnu par l'institution scolaire elle-même, puisque «faire connaitre à l'élève l'histoire de la construction de la connaissance scientifique est source d'inspiration pour la liberté intellectuelle $»^{7}$, mais privée de son contexte et de sa constitution d'origine, l'expérience historique perd les dimensions épistémologiques initiales qui lui ont donné suffisamment de sens pour traverser l'histoire ${ }^{8}$. La mise en place de réplication de ces mêmes expériences, respectant ces points, doit permettre d'aborder, outre les contenus déjà visés, les caractéristiques fondamentales de l'élaboration du savoir scientifique.

Répliquer une expérience historique nécessite de mettre en œuvre une démarche spécifique, qui s'éloigne beaucoup de la simple reproduction contemporaine d'un travail ancien. Cela nécessite en effet de respecter l'ensemble des conditions expérimentales de l'époque à laquelle l'expérience originelle a été menée. Il faut essayer de rester au plus près des outils, des moyens et des méthodes disponibles à l'époque. Nous cherchons donc à utiliser autant que possible des instruments de mesure de l'époque, afin de nous affranchir des effets d'intégration ou d'interprétation que pourraient développer des appareils de mesure modernes. Mais un instrument ancien, même s'il permet d'effectuer des mesures n'est pas nécessairement dans son état d'origine. Les données qu'il produit peuvent être le fruit d'une altération du temps sur ses constituants. Son usage nécessite alors, avant même de l'utiliser pour l'expérimentation, d'en tester le comportement pour le comparer à celui attendu par son fabricant à l'époque, lorsque ces données peuvent être disponibles. Ces instruments n'étant bien sûr pas toujours disponibles, ou n'étant pas dans un état permettant de réaliser des mesures fiables, nous pouvons être conduits à utiliser des appareils plus récents. Il nous faudra alors choisir des appareils aux principes de fonctionnement identiques à ceux des instruments d'époque, en en modifiant parfois la structure interne pour se rapprocher du fonctionnement d'origine. Cette contrainte est d'autant plus importante que certains auteurs ont mis en évidence le rôle que peut jouer l'instrument lui-même dans la manière de recueillir les résultats et de les analyser ${ }^{9}$.

Au-delà de l'usage de ce type d'instruments, il faut se garantir de la qualité des matériaux utilisés pour l'expérience. Si cette question semble possible à régler pour la plupart des expérimentations de physique, Elle l'est souvent beaucoup moins dès que l'on explore l'expérimentation chimique, dans laquelle nous n'avons que très rarement accès aux compositions chimiques exactes des réactifs utilisés.

Lors de la mise en œuvre de ces expérimentations sous l'angle de la réplication historique il est fondamental, outre l'usage d'instruments aux principes de fonctionnement semblables à ceux d'origine, de chercher à utiliser des modes d'enregistrement des résultats identiques. En effet, les processus d'acquisition modernes peuvent avoir un effet sur l'interprétation que nous faisons du fait expérimental. Le contexte d'enregistrement de ces données créé, comme l'ont montré plusieurs auteurs, des contraintes spécifiques à l'expérimentation qui conditionnent le nombre de tests que l'on peut mener, les délais nécessaires entre plusieurs tests et donc la multiplicité des données expérimentales exploitables lors d'une phase d'analyse.

Dans le cas de la production de rayons cathodiques, première étape vers celle de rayons $\mathrm{X}$, par exemple, une fois les rayons obtenus et observables dans un tube de Crookes, il faut pouvoir les analyser et donc les enregistrer. Un système de caméra moderne peut être une première étape dans la mise en œuvre de la réplication, mais pour agir comme le faisait un expérimentateur du XIXe siècle, il faut se diriger vers un enregistrement photographique argentique. Cela signifie travailler dans la quasi obscurité

\footnotetext{
${ }^{7}$ Ministère de l'Education Nationale, de la Jeunesse et de la vie Associative, Programme de physique-chimie en classe de seconde générale et technologique, Bulletin Officiel spécial $\mathrm{N}^{\circ} 4$ du 29 avril 2010, p1.

8 Rieß F. (2007) Short history of the use of historical experiments in German physics lessons, in Peter Heering and Daniel Osewold (eds), Constructing scientific understanding through contextual teaching. Berlin, Franck \& Timme, pp 219-226.

${ }^{9}$ Franklin A. (1986) The neglect of experiment. Cambridge, Cambridge University press.
} 
pour que le phénomène lumineux seul impressionne la plaque, mais aussi déclencher l'exposition de celle-ci à un moment choisi, et essayer de retrouver ce moment à chaque cliché, pour avoir des résultats comparables. Le contexte expérimental en est nécessairement modifié.

\subsection{Savoir-faire anciens et contraintes modernes}

Il nous faut donc essayer de nous placer dans les conditions les plus proches de celles de l'époque pour ce qui concerne l'organisation même du laboratoire. Ceci nécessite souvent d'arrêter la réplication pour tenter de reconstruire des procédures de manipulation courantes à l'époque de l'expérience originelle. Ces habitudes, et ces savoir-faire, contextualisés à une période historique, sont généralement partagés par l'ensemble de la communauté scientifique. Ils ne nécessitent donc à cet instant aucune description ou explication dans les publications ou les textes faisant état du déroulement de l'expérience. Ceci conduit à la disparition de ces savoir-faire lors de la disparition des contextes qui le nécessitent. L'expérimentateur moderne se doit donc souvent de les retrouver et de les reconstruire ${ }^{10}$.

Il est donc nécessaire d'effectuer un travail historiographique permettant de construire le contexte scientifique de l'époque étudiée. Les étudiants prennent ainsi conscience des enjeux scientifiques, mais aussi du contexte social dans lequel les travaux étudiés se sont déroulés. Ils comprennent l'importance que peut revêtir une expérience ou une découverte au regard des controverses scientifiques en cours à ce moment-là. Il est courant qu'une expérience historique, relatée dans les manuels scolaires comme une évidence et une preuve claire d'une réalité scientifique ait été au moment de son élaboration discutée, remise en cause voire même rejetée. L'élaboration de ce travail de contextualisation ne peut se faire que par l'utilisation de sources primaires telles que les publications des auteurs de l'époque (articles, ouvrages, traités), les cahiers d'expérience lorsqu'ils sont disponibles, les catalogues d'instruments. Il peut aussi parfois être nécessaire de recourir à des ressources " humaines », en particulier lorsque nous butons sur une difficulté technique. Certains corps de métier, en dehors du champ scientifique académique, emploient des méthodes traditionnelles utilisées autrefois dans les laboratoires de recherche. Ces techniques ont pu alimenter les savoir-faire des chercheurs et se perdre par la suite dans le monde académique, pour ne persister que dans le champ de l'artisanat traditionnel. Nous avons pu par exemple, afin de réaliser des cuves en bois étanche contacter des tonneliers bourguignons ${ }^{11}$.

Il faudra ensuite prendre en compte les risques et dangers auxquels nous nous savons exposés actuellement, et dont les auteurs historiques n'avaient pas connaissance. L'usage de substances toxiques, néfastes à l'environnement, devra se faire en prenant en compte la dangerosité mais aussi le risque de pollution, et pourra donc nécessiter certaines adaptations du processus instrumental. Les risques électriques, auquel nous pourrions être exposés doivent être protégé par des systèmes de sécurité modernes que nous chercherons à placer à l'extérieur du système instrumental.

Lors de la production de rayons X, interceptés et enregistrés sur une plaque photographique, l'habitude expérimentale et le pittoresque des premiers mois après la découverte voulaient que l'expérimentateur se radiographie la main, comme le représente la figure ci-dessous (Fig. 1).

Les premières publications sur l'extrême dangerosité de cette pratique et sur les premiers traumatismes graves liés à ce geste apparaissent moins d'un an après la première publication de Röntgen ${ }^{13}$ dans la revue la Nature, sous la plume de l'éminent physicien Charles-Edouard Guillaume, qui relate les mésaventures d'un démonstrateur en rayons $\mathrm{X}$ de l'exposition indienne de Londres durant

\footnotetext{
${ }^{10}$ Sur cette question, on pourra lire les travaux d'Otto Sibum : Sibum, H. O. (1998). Les gestes de la mesure : Joule, les pratiques de la brasserie et la science, Annales: Histoire, Sciences Sociales, 53(4-5), 745-774.

${ }^{11}$ Fatet J. (2005) Recreating Edmond Becquerel's electrochemical actinometer, Archives des Sciences, n58, pp 147-156.

12 Niewenglowski G.-H. (1897) La Photographie et la Photochimie, p. 235.

13 Röntgen W. (1895), Über eine neue Art von Strahlen, Comptes-rendus des réunions de la Société physico-médicale de Würzburg du 28 décembre 1895.
} 


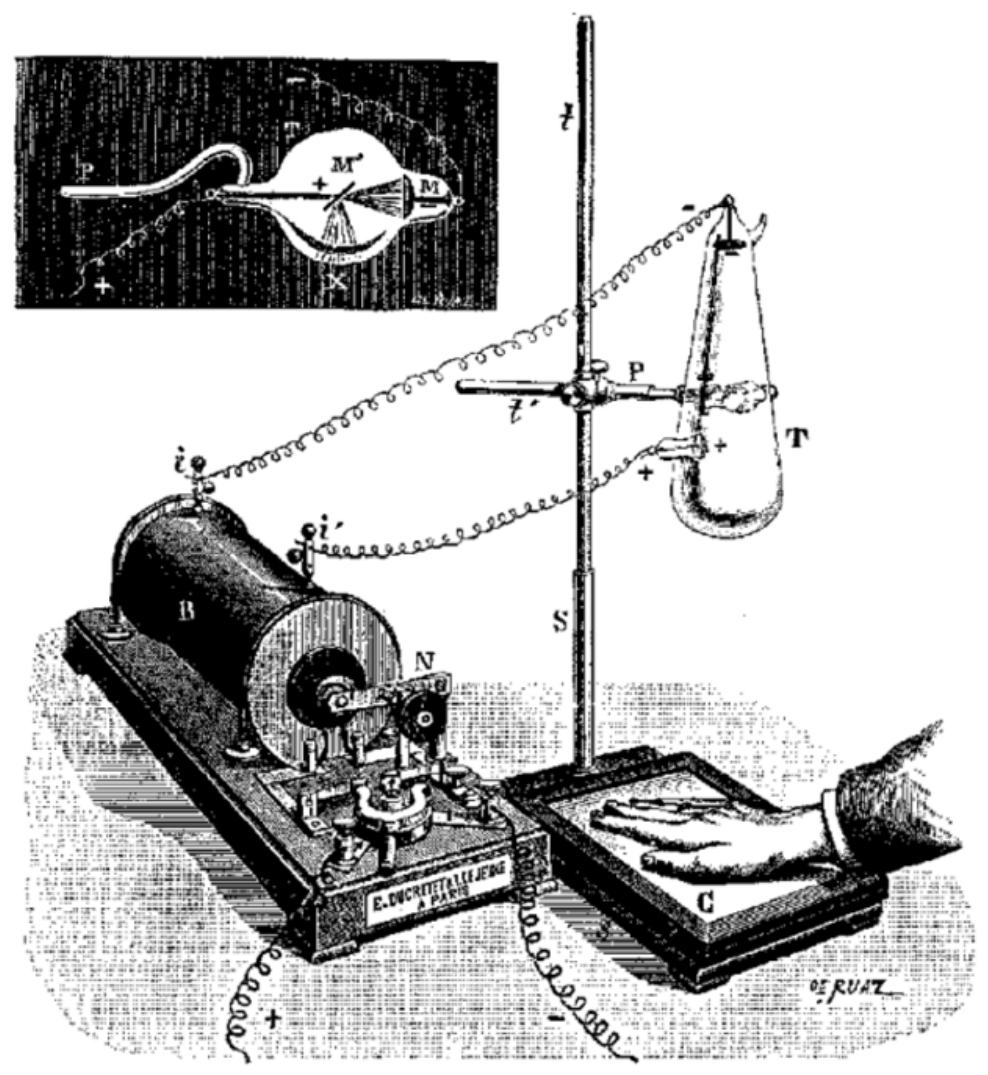

Figure 1. L'expérience de Röntgen, gravure extraite de G.-H. Niewenglowski ${ }^{12}$.

l'été $1896^{14}$. Il est évident que nous devons, lors de la réplication, nous protéger, et protéger les étudiants en nous tenant éloignés de la source, protégés par des tabliers et ne radiographier que des objets inertes : Ici (Fig. 2) la main de notre squelette de démonstration pédagogique :

L'ensemble de ces adaptations, la prise en compte des dangers, de l'environnement, de la qualité instrumentale des appareils anciens, limite l'exactitude de ce que l'on peut nommer réplication au sens où l'on souhaiterait produire des expériences parfaitement identiques à celles de l'époque. D'autres facteurs, tels que la grande difficulté à maîtriser les gestes et savoir-faire des expérimentateurs anciens, dont la virtuosité nécessitait des années de pratique, rendent difficile la précision et l'exactitude de la réplication.

La production de l'image photographique du phénomène étant le seul moyen d'identifier l'émission de rayon $\mathrm{X}$, on ne sait si l'expérience a fonctionné qu'en révélant la plaque photographique. Le temps nécessaire pour les réglages, les tests et la multiplication des résultats est donc démultiplié, puisque l'expérimentateur ne peut jamais affirmer que l'expérience en cours est efficace.

Toutefois toutes ces difficultés étant prises en compte, il est important de rappeler que notre objet ici n'est pas de reproduire des résultats anciens afin de les vérifier, ce qui serait scientifiquement inutile car totalement anachronique ou de critiquer la qualité des expérimentateurs de l'époque, mais bien d'explorer les enjeux épistémologiques d'une expérience, de sa discussion et de sa validation. Sous

14 Guillaume C.-E. (1896), Les méfaits des rayons X, La Nature n¹226 du 28 novembre 1896, pp406-407. 


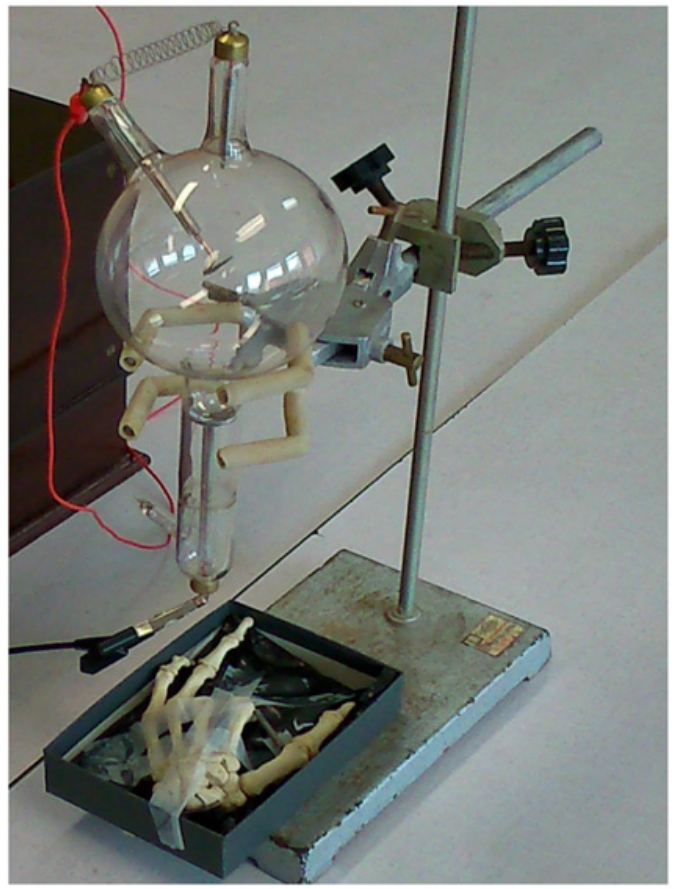

Figure 2. Main d'un squelette sous le tube à Rayon X.

cet angle, découvrir, comprendre, et contourner toutes ces difficultés et ces contraintes, apporte des informations et des connaissances permettant aux étudiants expérimentateurs d'élaborer par la pratique des savoirs épistémologiques que nous espérons employables dans leurs pratiques pédagogiques.

\subsection{Travail demandé et production des étudiants}

Concrètement, les étudiants concernés sont, au début de l'année, confrontés à un fait expérimental que nous pensons réplicable dans notre laboratoire, seuls ou en binôme. Nous les accompagnons dans son exploration bibliographique et la recherche de sources primaires. Lorsqu'ils considèrent qu'ils disposent de suffisamment d'informations pour commencer un travail de réplication, ils construisent un protocole précis, détaillant en particulier les différentes phases d'introduction des éléments expérimentaux originels si cela est nécessaire : production du phénomène, organisation de l'espace, sources d'alimentation, appareils d'enregistrement par exemple. Les étudiants disposent alors d'un espace permanent dans lequel leur montage expérimental pourra être conservé et évoluer au cours de l'année. Le passage de chacune de ces étapes s'accompagne d'une activité d'analyse technique et physico-chimique qui la justifie, ainsi que d'arguments issus des sources anciennes disponibles. Chaque étape s'achève par une analyse réflexive des processus expérimentaux mis en jeu, des difficultés rencontrées, des choix qu'il a fallu faire pour les contourner ou les résoudre. La fin de la réplication, que les étudiants pensent atteindre en obtenant les mêmes résultats que les expérimentateurs anciens, est souvent décidée lorsqu'il «n'est pas possible faire mieux ». Nous présentons en Fig. 3 l'une des images produite par exposition aux rayons $\mathrm{X}$ des ossements mentionnés plus haut, et enregistrée sur une plaque photographique placée dans une chambre photographique du début du XXème siècle. Elle a pu être obtenue après plusieurs mois de réplication. 


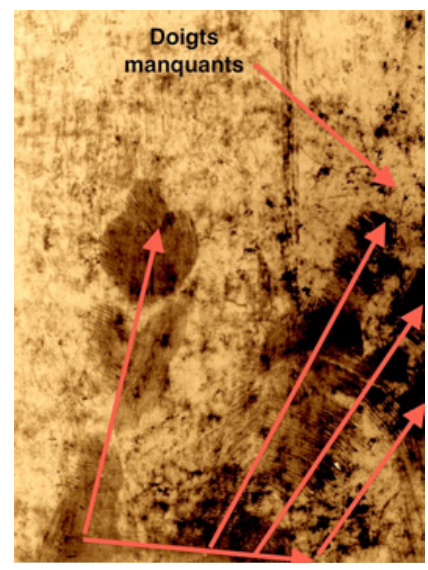

Figure 3. Image sur plaque photographique exposée aux rayons $\mathrm{X}$. Les flèches indiquent les directions des ossements radiographiés.

La production finale est un rapport détaillant le contenu historique, puis le projet expérimental, et enfin les résultats analysés de la réplication. Lorsque les contenus s'y prêtent, ce document peut être complété par une mise en relation avec les programmes scolaires actuels et une possible utilisation de ce travail de réplication dans le contexte de l'enseignement secondaire. Les étudiants sont accompagnés, tout au long de ce processus par un enseignant-chercheur compétent sur les contenus historiques et scientifiques.

\section{Apports épistémologiques}

\subsection{Apports pour les étudiants scientifiques}

Les expérimentations que nous menons portent sur différents champs de la physique et concernent plusieurs époques de l'histoire des sciences. Bien qu'il soit difficile de recueillir des données exploitables et analysables en termes de connaissances et de compréhension de l'épistémologie pour les étudiants ayant participé à cet exercice, il semble qu'un certain nombre d'objectifs d'apprentissage ont été atteints. D'un point de vue purement historique les étudiants ont été amenés à élaborer ou expliciter des données historiques nouvelles que seule la réplication pouvait permettre d'imaginer concernant la méthode de fabrication de certains appareils, les méthodes de recueil et de tri de données expérimentales, et les méthodes d'enregistrement et de conservation de ces données et de ces faits. Cela leur a permis de construire des liens entre des domaines qui pour eux restaient parfaitement distincts du champ des sciences expérimentales, et de la physique en particulier. En effet, dans le contexte de l'expérimentation, ils ont pris conscience de la nécessité de maîtriser simultanément plusieurs champs théoriques de la physique pour parvenir à réaliser une simple série de mesures, qui pourtant paraissait évidente avant la réalisation.

Produire des rayons X par exemple, nécessite de maîtriser les questions liées à l'émission des rayonnements bien sûr, mais aussi des questions d'électricité, d'induction et de flux, puisque notre tube est alimenté par une bobine de Ruhmkorff. Cette bobine étant alimentée par une pile au bichromate, la chimie de l'oxydoréduction est aussi indispensable. Enfin, l'enregistrement des résultats se fait sur une plaque photographique, ce qui nécessite des connaissances en photochimie et en chimie photographique.

Les étudiants ont été amenés, par la simple nécessité de leur travail, à s'interroger sur les processus d'élaboration des résultats expérimentaux. Il leur a fallu explorer les différents rôles que peut jouer la théorie dans l'obtention et l'exploitation de données expérimentales. En effet, la justification théorique 
historique d'une expérimentation et de ses résultats est souvent différentes de celles qu'on apporterait actuellement. Cette justification théorique participe aux choix techniques qui peuvent être faits pour l'élaboration de l'expérience ${ }^{15}$. Les étudiants sont alors amenés à réfléchir au sens que peut avoir le fait de présenter ses expériences comme «preuve» de la théorie actuelle. Ils sont de plus amenés à étudier les processus de validation ou de rejet d'une expérience ou d'un résultat expérimental en prenant en compte le contexte socio-scientifique d'une époque. Le rôle et la place des controverses dans ces processus de validation, et dans la nécessité même de mettre en œuvre certaines expérimentations comme argument du débat, éclairent d'une lumière différente la question du vrai et du faux, et de la preuve comme critère froid et objectif de la connaissance scientifique.

On voit aussi apparaître chez les étudiants mettant en œuvre ce type de travaux des réflexions sur la qualité, la finesse, la précision des expérimentateurs de l'époque. Ils découvrent de plus l'excellente qualité de la plupart des appareils de mesure, parfois bien meilleure que celle des multimètres numériques en lesquels ils avaient pourtant une confiance aveugle. Cette mise en perspective, qui construit l'image d'une science déjà performante et efficace, parfois plus précise que celle mise en œuvre dans l'enseignement d'aujourd'hui, apporte beaucoup à la compréhension d'une science en train de se faire, en discussion, en élaboration.

Lors de l'apparition de ces questionnements, que nous pouvons suivre en accompagnant les étudiants dans la réalisation sur le long terme de ces séances de réplication, il nous est alors possible d'introduire de manière formelle et précise les concepts épistémologiques qu'ils viennent de «toucher du doigt ». Nous pouvons alors rattacher leur nouvelle compréhension des caractéristiques spécifiques de la physique en tant que science expérimentale aux réflexions d'auteur, et ainsi les ancrer dans le champ académique de l'épistémologie des sciences. L'introduction de ces concepts fait alors sens puisque les étudiants ont expérimenté, au sens concret du terme, ces concepts épistémologiques. Ils prennent corps, ils ne sont plus de simples réflexions, parfois vaporeuses, sur l'élaboration des sciences mais des outils utiles à la pratique, à la compréhension des concepts et à la délimitation des modèles.

\subsection{Apport à la formation des enseignants}

Les étudiants que nous formons se destinent à devenir à très court terme enseignants de sciences physiques. Nous avons pu aussi expérimenter ce processus dans le cadre de formation continue d'enseignants du second degré. Les apports épistémologiques pour la formation des enseignants et l'efficacité d'un enseignement renouvelé des sciences, en accord avec les programmes, semblent significatifs. D'une part, les concepts épistémologiques fondamentaux communément partagés par la communauté des chercheurs en physique, tel que le concept de falsifiabilité, les caractéristiques fondamentales liées à la reproductibilité des expériences et à la transmission des résultats, souvent obscures ou purement théoriques, lorsque l'on n'a pas eu l'occasion de s'exercer à la pratique en laboratoire, prennent sens. Cela permet de rediscuter du rôle et du statut de l'expérience dans l'élaboration du savoir scientifique et donc de réfléchir à ceux-ci dans les processus de transmission et d'acquisition de ces mêmes savoirs. La question du sens que l'on peut donner à l'expression « de preuve expérimentale » est très rapidement soulevée. Cette question, fondamentale dans le contexte de l'enseignement des sciences physiques, est ici abordée en permettant aux enseignants de réfléchir à l'usage qu'ils en font dans la classe. Le sens de la séance de travaux pratiques traditionnelle, application directe d'un cours théorique, servant à asséner la certitude que ce que l'on vient d'apprendre est vrai, est remis en question. Le travail qu'ont réalisé les étudiants concernant l'impact du contexte historique dans l'élaboration et l'exploitation de l'expérience, ainsi que dans l'obtention et le tri des résultats, leur

15 Galison P. (1987) How experiments end, Chicago University press. 
permet de s'interroger concrètement sur la question du vrai, du vraisemblable, et donc sur le champ d'application et le sens des modèles manipulés.

Ces questions sont extrêmement prégnantes dans l'enseignement des sciences actuellement puisque de nombreux processus sont mis en œuvre, telle que la démarche d'investigation et les démarches actives d'une manière générale, visant à mettre l'élève " dans la position du chercheur ». L'expérimentation menée avec nos étudiants, qui permet de les mettre, toute mesure gardée, dans la position d'un chercheur ayant vécu, les amène à relativiser la possibilité de construire des situations dans lesquelles les élèves se trouveront dans ce contexte. Les questions socio-scientifiques et le contexte technique d'une époque jouant un rôle majeur, tel qu'ils l'expérimentent, sur l'élaboration même de l'expérience et sur la relation qu'ont expérience et théorie à un instant donné. Cela semble dès lors très difficile à construire avec les élèves lors d'une démarche d'investigation.

\section{En guise de conclusion, quelques perspectives}

Les établissements scolaires du second degré possèdent souvent un fond d'instruments anciens, datant majoritairement des dotations la IIIe République, dont la conservation, lorsqu'elle parvient à ne plus être un problème, n'est réalisée qu'à des fins la plupart du temps purement esthétiques. On nettoie, on fait briller, et on met en vitrine des appareils anciens dont l'objectif premier était pourtant scientifique et non esthétique. Notre projet est de proposer à terme un fond d'expériences historiques réplicables à l'aide du matériel disponible dans la plupart des établissements du second degré, ou ne nécessitant que peu de bricolage, afin de permettre aux enseignants d'acquérir dans le cadre de la formation continue, les compétences pour réaliser ce travail avec leurs élèves. Choisissant des points du programme particulier, il semble en effet possible de proposer cette démarche comme mode de réflexion sur le sens de la pratique scientifique et du savoir scientifique lui-même, tout en créant des ponts entre différents domaines de la physique (électricité, rayonnement, mécanique, ...) et entre différentes disciplines d'enseignement trop souvent cloisonnées.

Au-delà d'un effet possible de ces travaux dans le cadre de la lutte contre la désaffection des sciences, sur laquelle on doit continuer à s'interroger, ceci permettrait de développer avec les élèves une nouvelle approche des savoirs scientifiques orientée vers la réflexion sur leurs validités, leurs limites, leurs modèles, qui participeraient de façon certaine à la construction du citoyen du XXIe siècle.

\section{Références}

J. Fatet (2005) Recreating Edmond Becquerel's electrochemical actinometer, Archives des Sciences, n 58 , pp 147-156.

A. Franklin (1986) The neglect of experiment. Cambridge, Cambridge University press.

P. Galison (1987) How experiments end, Chicago University press.

C.-E. Guillaume (1896), Les méfaits des rayons X, La Nature $n^{\circ} 1226$ du 28 novembre 1896, pp406-407.

P. Heering, R. Wittje (2011) Learning by doing, Franz Steiner Verlag.

T. Kuhn (1983) La structure des révolutions scientifiques, trad.. Laure Meyer, Champs/Flamarion $n^{\circ} 115$, p76.

Ministère de l'Education Nationale, de la Jeunesse et de la vie Associative, Programme de physiquechimie en classe de seconde générale et technologique, Bulletin Officiel spécial $N^{\circ} 4$ du 29 avril 2010, p1.

Ministère de l'Education Nationale, de la Jeunesse et de la vie Associative, Programme de l'enseignement spécifique et de spécialité de physique-chimie, Bulletin Officiel spécial $N^{\circ} 8$ du 13.10.2011, p1.

G.-H. Niewenglowski (1897) La Photographie et la Photochimie, p. 235. 
Les sciences humaines dans les parcours scientifiques et techniques professionnalisants

F. Rieß (2007) Short history of the use of historical experiments in German physics lessons, in Peter Heering and Daniel Osewold (eds), Constructing scientific understanding through contextual teaching. Berlin, Franck \& Timme, pp 219-226.

W. Röntgen (1895), Über eine neue Art von Strahlen, Comptes-rendus des réunions de la Société physico-médicale de Würzburg du 28 décembre 1895.

H.O. Sibum, (1998). Les gestes de la mesure: Joule, les pratiques de la brasserie et la science, Annales : Histoire, Sciences Sociales, 53(4-5), 745-774. 